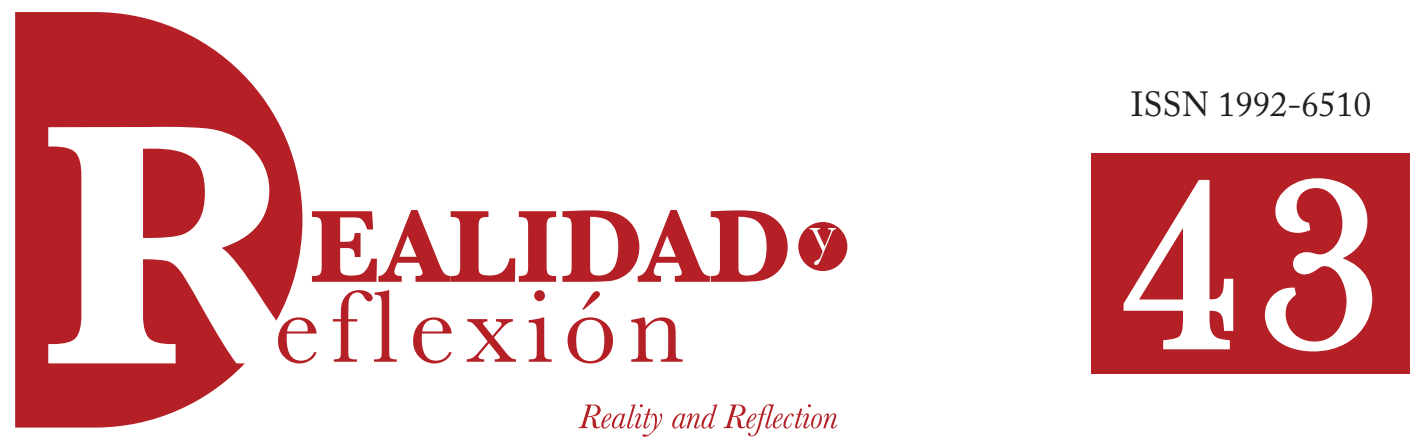

Año 16, N 43, San Salvador, El Salvador, Centroamérica. Revista Semestral Enero-Junio 2016

YEAR 16, N 43, SAN SALVADOR, EL SALVADOR, CENTRAL AMERICA. SEMESTRAL JOURNAL JANUARY-JUNE 2016

\title{
Estudiantes y maestros que utilizan los smartphones y otras tecnologías educativas en las aulas universitarias para mejorar el proceso de la enseñanza-aprendizaje
}

\author{
Students and teachers who use smartphones and other \\ educational technologies in university classrooms to improve \\ the process of teaching and learning
}

\author{
MEd. Fidel Arturo López Eguizábal \\ Catedrático de la Facultad Ciencias Económicas. \\ Investigador del Instituto de Ciencia, Tecnología e Innovación \\ de la Universidad Francisco Gavidia.
}

\section{RESUMEN}

La falta de motivación de parte de los catedráticos y la proliferación de los teléfonos inteligentes o smartphones son factores que provocan la falta de atención de los alumnos en clases. La distracción aumenta por el uso de estos teléfonos.

Esta investigación aborda la importancia de las nuevas tecnologías educativas que pueden ser utilizadas por los maestros, muchos de los cuales no las manejan por falta de interés o porque las universidades no se han detenido a observar su valor en el proceso de enseñanza-aprendizaje. Los alumnos son los que están conectados a Internet en el salón de clases, es por ello que se debe analizar el debido uso de esta red y sus consecuencias. El documento presenta los argumentos a través de una investigación cualitativa-cuantitativa sobre la utilización de artefactos tecnológicos en el salón de clases.

En bachillerato se han realizado investigaciones sobre la mala utilización de los teléfonos inteligentes u otros aparatos. En la universidad sucede lo mismo, solamente que en bachillerato u otros grados, hay más restricciones de parte de los maestros y las autoridades de las instituciones. En las aulas universitarias, en cambio hay más libertad, los estudiantes se conectan libremente a wifi, todo ello hace que sea facilita el acceso a internet.

La investigación se realizó en cuatro universidades, a saber: Universidad Francisco Gavidia, Universidad Tecnológica de E1 Salvador, Universidad Modular Abierta y Universidad de E1 Salvador.

Palabras clave: aprendizaje, tecnología, smartphone, redes sociales 


\section{ABSTRACT}

Students do not pay attention in class because of lack of motivation on the part of professors and another factor contributing to the neglect is the proliferation of smartphones. Distraction increases with the use of these phones.

The research is focused on addressing the importance of educational technologies, which can be used for the generation of teachers who are not yet aware of its use. Many do not handle them for lack of interest, because other universities have not seen the importance in the process of teaching and learning. Students are those who are connected to the Internet in the classroom, which is why we must analyze the proper use of this network and its consequences.

The arguments are also presented through a qualitative and quantitative research on the use of technological devices in the classroom. In high school they have conducted research on the misuse of smart phones or other devices. In college same thing happens, only that in school or other degrees, there are more restrictions on the part of teachers and the authorities of the institutions. In university classrooms, however there is more freedom, students are free to connect to wifi connections, all this makes it easier to connect.

The research was conducted at four universities: Universidad Francisco Gavidia, Universidad Tecnológica de El Salvador, Universidad Modular Abierta y Universidad de El Salvador.

Keywords: learning, technology, smartphone, social networks

\section{Introducción}

No podemos rechazar totalmente los avances que ofrecen las tecnologías educativas; es importante destacar que la utilización de aparatos educativos como los smarthphones (teléfonos inteligentes) ayuda a que la información sea más inmediata: las tareas sean investigadas con más interactividad, con mayor eficacia y eficiencia.

En El Salvador, ya nos encontramos con una nueva generación conocida como "la generación millennial o generación Y", es la subsiguiente a los “Gen Xers o Generación X”, de 18 a 30 años, nacidos entre las décadas de los 80 a los 90, hasta principios de la primera década del año 2000. "Las características de los millennials varían según la región y sus condiciones socioeconómicas... se les caracteriza por el uso masivo de las redes sociales y su 'familiarización' innata con la comunicación, los medios y la tecnología digital". ${ }^{1}$

La sociedad del conocimiento permite que en

1 http://www.leanstart.es/millennials-una-nueva-generacion/ 7 de agosto del 2015. las universidades se busquen nuevas formas de aprendizaje; el uso de los celulares ha venido a revolucionar al ser humano. En E1 Salvador casi todos sus habitantes poseen uno o más celulares.

Si ese aparato se usase para propósitos educativos o de investigación, seríamos un país educado. Sin embargo, seguimos siendo un país que copia culturas y estereotipos. El tener un celular es nada más que todo un negocio de las compañías y el aparato es para el usuario como su propia madre o ser amado.

En el salón de clases, todo el mundo evidencia que muchos están entretenidos utilizando sus celulares. "Parece que están orando", dijo alguien, ya que al estar inclinados inician sus conversaciones.

Algunos se ríen, parece que están locos o que se han ido en cuerpo y en alma a un lugar recóndito. En plena clase, el maestro le hace una interrogante al alumno que habla por celular, 
y éste se despierta y daba la impresión de que estaba en un estado hipnótico. Su lenguaje no verbal evidencia que estaba en un "shock"; esto debido al celular; aparato que vino a quedarse en la vida de los estudiantes. Es más, si se le dice que "ponga atención", hasta se molesta y es capaz de decirle un par de cosas al docente.

La presente investigación se hace con el objetivo de romper el paradigma de que al utilizar los celulares en el aula se logra un fin educativo en el sentido de obtener mejores resultados en el aprendizaje. No se abordan solo aspectos negativos, se debe tomar en consideración que hasta en centros de educación media, como "ABC Bilingual School, utilizan tablets y otros aparatos para el aprendizaje de sus alumnos. Desde pequeños, los estudiantes se acostumbran a interactuar con la tecnología, saben bien los maestros de la importancia al utilizarla para el bien de los alumnos. En el plan de estudios se incluye "altamente tecnológica y uso de IPADS desde parvularia”. ${ }^{2}$

Las Tecnologías de la Información y las Comunicaciones (TIC) permiten que en cualquier nivel académico las tecnologías educativas, en espacial los celulares, ayuden a ser más interactivas las clases, contribuyen a que el maestro indague y sea más amena la conversación, y por ende el aprendizaje.

En el ámbito universitario la responsabilidad de los maestros y padres de familia es diferente; es responsabilidad de los alumnos saber utilizar correctamente el teléfono inteligente en el salón de clases. Existen alumnos responsables, quienes hasta piden permiso para poder revisar su celular

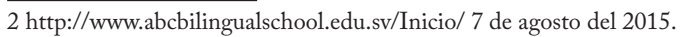

para consultas del tema, al final para estos, la clase se vuelve más constructiva e interactiva.

¿Está el maestro innovando en el salón de clases o se ha quedado en la era de la pizarra y el yeso? ¿Puede mezclar lecturas entre libros físicos e información virtual para el proceso enseñanza-aprendizaje?

\section{Metodología utilizada}

La investigación está dividida en cuatro partes esenciales, la primera inicia con un video que determina la importancia de tener un celular inteligente para indagar el significado de “¿qué es paradigma?, en la cátedra de Ciencias de la Comunicación II. La interrogante planteada por el catedrático fue abordada por todo el alumnado en el salón de clases.

En la segunda parte se preguntó a los estudiantes, a través de la red social Facebook, si es favorable o dañino el uso del celular en el aula.

En la tercera parte de la investigación se realizaron entrevistas a catedráticos, los cuales tienen la batuta de permitir o no el uso de los celulares $u$ otro aparato tecnológico en el aula. ¿Es cultural la utilización de los celulares inteligentes en el salón de clases o son las currículas educativas modernas las que permiten tal utilización?

Por último, se hizo una encuesta con una muestra aleatoria de 178 cuestionarios con 10 preguntas, en donde se destaca la utilización positiva y negativa de los teléfonos inteligentes, se graficaron y analizaron los resultados, con el propósito de tener una idea de lo bueno y lo malo de la utilización de tecnologías educativas. Un alcance significativo para identificar los 
aspectos que ayuden a comprobar si ayuda o no al proceso de enseñanza-aprendizaje se propone esta investigación.

\section{¿Por qué evitar el celular en clases?}

Se ha comprobado que la mayoría de alumnos que utiliza sus celulares en clase para cualquier propósito, dejan el curso al final del ciclo o aplazan la asignatura. Es difícil que el cerebro se concentre estando en el aula, utilizando el celular y poniendo atención en la clase. Solamente si con este aparato se hace una actividad académica, se podrá aprender, y si existe una pausa para utilizarlo sería recomendable su uso.

En la conclusión del estudio "El uso de teléfonos móviles en el sistema educativo público de E1 Salvador: ¿Recurso didáctico o distractor pedagógico?”, el investigador Herberth Alexander Oliva, manifiesta que: “...Se obtuvo una tendencia a encauzar el celular como el principal enemigo actual del aprendizaje (...) los estudiantes prefieren dar prioridad a la tecnología (...) que a lo que se explica en clases". ${ }^{3}$

Lo positivo se retoma del siguiente concepto: "Se debe diseñar una estrategia didáctica para integrar el uso de celulares como un medio alfabetizador funcional para tecnologías (...) Hoy más que nunca, vemos una gran cantidad de alumnos utilizando las aplicaciones de los dispositivos móviles inteligentes para llevar a cabo sus tareas, tanto de trabajo o de estudio como de entretenimiento por medio de internet" (Franco, 2008).

3 Oliva, Herberth Alexander. (2015). "El uso de teléfonos móviles en el sistema educativo público de El Salvador: ¿Recurso didáctico o distractor pedagógico?”, revista Realidad y Reflexión, Universidad Francisco Gavidia, julio diciembre 2014.4, Ibídem.
En el ámbito universitario, y como se mencionó, las reglas son distintas, pues cada alumno es libre de entrar al aula a la hora que desee, dependiendo de la asignatura y del catedrático. Esto, como primer punto para que los estudiantes entren y luego manipulen sus celulares. Algo que es común en los seres humanos que están al unísono con las tecnologías. En el salón de clases los alumnos interactúan, lo que no se puede evitar. Lo que se pretende es que utilicen los celulares para investigar. La comunicación de los estudiantes es permanente en el aula, principalmente en los que se sientan atrás, en donde hay alumnos que usan el celular para mensajear, textear o aun escuchar música.

En otras investigaciones, en educación media se constata lo siguiente:

Dave Stovenour, vicedirector de la Secundaria Dundalk en Baltimore, concuerda con que es necesario capacitar a maestros y alumnos en el uso de dispositivos móviles. Para él, la respuesta no es prohibir el uso de celulares, sino enseñarles a los niños cuándo usarlos.

"Nos dimos cuenta de que también hace falta enseñarles a los alumnos los usos apropiados", dijo Stovenour en la Asociación para la Supervisión y el Desarrollo de Planes de Estudio. "Hoy en día, sigue estando prohibido usar el celular en el aula, pero se permite en la cafetería o en los pasillos... se les recuerda los usos apropiados. Observamos una caída en el número de interrupciones causadas por dispositivos electrónicos, y los padres recibieron bien esta política más relajada”.

En el ámbito universitario la situación es diferente: no se puede prohibir el uso de los celulares a los alumnos. Es muy difícil que dejen ese aparato; pues 
prácticamente es parte de sus vidas. La "Generación Y”, como se mencionó, está por doquier.

En el salón de clases se experimentó, a través de una pregunta dirigida por el maestro, si todos utilizaban debidamente el celular o teléfono inteligente $\mathrm{u}$ otro aparato que sirviese para consulta educativa. La interrogante fue: ¿Qué es paradigma? Luego, todos iniciaron la búsqueda de la respuesta y se constató que algunos estaban en "Whats $A p p$ " u otra forma de comunicación.

Se confirmó que fueron pocos los que realizaron otra actividad que no sea educativa en la interrogante planteada.

Se demostró que si el maestro supervisa a los alumnos, se podrán obtener resultados satisfactorios; empero, hay maestros a quienes no les interesa el aprendizaje de los alumnos, más cuando el aula está abarrotada con más de cien estudiantes. Es difícil entonces mantener el control. Los alumnos que están sentados atrás, en su mayoría están conectados a Internet, pero no para aprender. Se puede observar el ejercicio realizado para esta investigación en el vídeo en YouTube en el siguiente enlace: https://www. youtube.com/watch?v=rZOXT_MUlzE



Fuente: video creado y subido a la plataforma YouTube por el investigador. Disponible en: https://www.youtube.com/watch?v=rZOXT_MUlzE

\section{Aspectos positivos y negativos en la utilización de los celulares y otras plataformas electrónicas}

Interactividad es lo que desean los alumnos si se comprueba que almacenar las clases en la plataforma virtual (U Virtual), hace perder el interés en el aprendizaje.

Unas de las reglas que se advierten en el aula son estas: no coma, no utilice celular, no fume, etc. La que nos interesa y es el objeto de estudio es la regla de "las tecnologías educativas". Para ello es necesario que los estudiantes y profesores se acoplen a las Nuevas Tecnologías de la Información y las Comunicaciones, que son estas:

- El aprendizaje es más interactivo.

- El maestro tiene una herramienta de apoyo rápido y eficaz para investigar en el salón de clases.

- Los alumnos investigan más rápido.

- Se logra hacer más amena la clase, no importa el tipo de cátedra.

- Los maestros también tienen que poseer teléfonos inteligentes para interactuar e indagar.

- Interés de parte de los alumnos "Generación Y” en aprender más.

"A pesar de todo, la solución no es prohibir los celulares ni las calculadoras en el salón de clases, sino incorporarlos en el proceso dinámico de educación... Debe haber una mezcla de exposición formal de un tema, con la actividad informal de discutir, descubrir, entender $y$ presentar un proyecto, lo que refuerza un tema en cuestión”. ${ }^{4}$

Es de hacer notar lo positivo al utilizar celulares:

\footnotetext{
4 Franco, J. (2008). Educación y Tecnología: solución radical. México: Siglo XXI; p. 290.
} 
en mi experiencia como catedrático, los alumnos no entran a clases por no considerar atractiva la clase, punto que difiere con argumentos o contraposiciones como el de utilizar o no un celular u otro artefacto en el aula.

En el Cuadro $n^{\circ}$. 1 , se muestran opiniones de siete estudiantes de la Universidad Francisco Gavidia, de la red social Facebook, sobre la interrogante ¿Por qué razón en clases los alumnos están viendo su smartphone en vez de poner atención?

Lo anterior muestra que la mayoría de estudiantes consultados está de acuerdo con que se utilice los celulares; pero con el propósito de investigar acerca de la clase. Si los alumnos siguen perdiendo el tiempo, podrían aplazar la asignatura. La opinión de los estudiantes consultados indica aquí que los celulares se usan con fines de investigar sobre la clase.

En colegios u otras instituciones de educación media, las autoridades no permiten el uso de celular. Al entrar al centro escolar, los alumnos dejan su celular en una “caja”. Con ello se está coartando la libertad de utilizarlo. Pero si los alumnos fueran conscientes podrían utilizar el celular para fines de investigar.

Los alumnos pueden llevar la mejor tecnología al aula, fingiendo que están conectados, investigando en Internet, mientras el maestro imparte la clase; pero los alumnos están en otro mundo con sus celulares funcionando, así de simple; para verificar este fenómeno baste con visitar un salón de clases.

En otro ámbito, un salón de clases sin tecnologías indica que se continúa con el sistema antiguo de la tiza y la pizarra, en donde no hay interrupciones, pero tampoco hay interactividad. Todos deseamos clases constructivistas. ¿Se debe entonces, permitir el uso de los celulares en el aula sin restricción alguna?

\section{La evaluación de competencias educativas}

"Peón, Anaya y Olguín (2000) hacen referencia a la incorporación de salones multimedia en las universidades. Un salón multimedia lo interpretan como el espacio físico en el que hay acceso a dos o más medios como la televisión, la computadora, el audio o el texto para propósitos educativos". ${ }^{5}$

Los salones con toda la gama de tecnologías educativas vuelven más eficaz un tema específico, y si es necesario se pueden emplear videos, audios u otro tipo de ayuda didáctica. Un salón con tecnologías educativas no es la panacea para resultados óptimos; empero, ayuda a la interactividad y la comunicación. Contribuye a que los alumnos tengan más posibilidades de investigar, de buscar información de interesarse por la clase.

Por otro lado, gran parte de los trabajos en este contexto han sido focalizados en el uso de las TIC bajo ambientes virtuales o de educación a distancia. Ibáñez (2004) menciona que hay muy pocos estudios experimentales que abordan objetivamente su impacto en el aula, solicitando trabajos sin sesgos oportunistas sobre el tema de la mejora de la calidad educativa a través de estas tecnologías. Ramírez (2001, en Organista y Backhoff, 2002) indica

5 “Tecnología educativa en el salón de clase. Estudio retrospectivo de su impacto en el desempeño académico de estudiantes universitarios del área de Informática”. Román Martínez Martínez; Yolanda Heredia Escorza, Revista Mexicana de Investigación Educativa 2010, 15 (45) p.374. 


\section{Cuadro $\mathrm{n}^{\circ} .1$}

\section{¿Por qué razón en clases los alumnos están viendo su smartphone en vez de poner atención?}

\begin{tabular}{c|l}
\hline Nombre del alumno & \multicolumn{1}{c}{ Opinión } \\
\hline Tatiana Domínguez & En el teléfono, a veces bajamos las diapositivas. \\
\hline Irvin Vides M. & $\begin{array}{l}\text { Para mí, es la falta de compromiso del estudiante, ya que realmente su mente } \\
\text { no ha madurado como estudiante; prácticamente llega con la misma actitud } \\
\text { del bachillerato y no ha fijado realmente el compromiso de la atención hacia } \\
\text { el docente: he ahí el problema. }\end{array}$ \\
\hline
\end{tabular}

Falta de interés sobre la clase... cuando algo te interesa está pendiente de ello, sea lo que sea, y cuando no, simplemente uno se vuelve más vulnerable a la distracción o uno mismo la busca por medio del teléfono.

Adriana Arrieta

Muchas veces eso es problema del estudiante, porque estudia solo por compromiso y por tener un título; por ende no le importa la clase... sin embargo, hay catedráticos que no explican bien y no se dan a entender, entonces se pierde "el hilo" de la clase y se hace presente en teléfono para "pasar el rato" en la clase.

En la Gavidia, y por experiencia propia, cuando las clases son de más de 40 o 50 personas, y los profesores no tienen micrófono y las otras herramientas, Henry Pineda es más difícil poner atención y por eso uno se distrae, y es más tentador ocupar el teléfono. Dependiendo de algunas clases, también se puede andar algún material de consulta en el celular.

Podríamos decir varios factores: primero, falta de interés en el aprendizaje y falta de educación; segundo, a veces hay docentes que carecen de pedagogía, y esto hace que se pierda la atención. Otras veces, porque el estudiante cree que hay materias que son solo para pasarlas y no para aprender; y la última, la menos posible, que el celular les sirva de herramienta para la comprensión de la clase.

Pero los alumnos pondríamos atención si el sistema de educación fuera eficiente. El ser humano, naturalmente, posee deseos de aprender; sin

Carlos Argumedo embargo, cuando no se sustenta de manera adecuada se aburre y se estresa, y consiguientemente busca una distracción.

Francisco Martínez

Una caja para depositar el celular en la entrada del salón de clases sería una solución simple y efectiva, ¿no lo cree?

Fuente: Interrogante realizada en red social en Facebook de Fidel López Eguizábal (marzo del 2015).

que sólo $10 \%$ de las investigaciones realizadas en México sobre este tema hasta 2002 hablan del uso de la computadora en el salón de clases y la actitud positiva que han tenido los alumnos ante la presencia de las TIC, enfatizando que son pocas las que miden el impacto en el rendimiento escolar... ${ }^{6}$

6"Tecnología educativa en el salón de clase. Estudio retrospectivo de su impacto en el desempeño académico de estudiantes universitarios del área de Informática”. Román Martínez Martínez; Yolanda
No podemos, en pleno siglo XXI, obviar las tecnologías educativas, pues hasta en las parvularias, los maestros enseñan con laptops a los niños. Por lo tanto, en otros niveles educativos $\mathrm{y}$, por ende, en la universidad, los teléfonos inteligentes son una herramienta útil para aprender y enseñar. Eso, según mi experiencia

Heredia Escorza, Revista Mexicana de Investigación Educativa 2010, 15 (45) p.376. 
docente. Las TIC son un gran avance para el aprendizaje moderno.

Las competencias educativas no son solamente para que los alumnos las utilicen; es imprescindible tecnificar y capacitar, tanto a maestros y alumnos, en el buen uso de todo aparato que sirva para el aprendizaje, tanto en el aula como fuera de esta.

¿Cómo regular el mal uso de los smartphones en el salón de clases?

El mal uso de las tecnologías educativas está implícito en la regulación del centro de estudios y del mismo maestro. No podemos estar a cada instante diciéndole al alumno que deje el celular. En esta realidad de la sociedad del conocimiento, todos poseen uno o más celulares en sus manos.

A continuación se presentan opiniones de maestros, acerca de la utilización de los smartphones $\mathrm{u}$ otros aparatos en el aula:

¿Usted está de acuerdo en que los alumnos utilicen celulares en las clases? ¿Cuál es su comentario al respecto?

\section{Lic. Mario Ruiz Aguilar, Director de Maestrías} Universidad Francisco Gavidia. "Estoy totalmente de acuerdo, ya que son un medio a través del cualla información está más inmediata y a la mano. La tendencia a la mEducation (es una organización comprometida a disminuir las desafíos que existen para acceder a tecnologías móviles adecuadas, flexibles y de bajo costo) es una realidad, aunque aún es poco entendida y no en todos los países se está aplicando de la mejor manera, por el rechazo por parte de algunos docentes, por desconocimiento de las herramientas disponibles para ello y por falta de capacidades de manejo de grupos usando esta tecnología (aspecto pedagógico). Quienes están en contra de esto, argumentan que el teléfono móvil es un distractor; no obstante, humildemente opino que lo será de la misma forma que lo sería cualquier otro artículo (tecnológico o no). También considero que un móvil será distractor en la medida en que la clase del profesor no sea interesante; es decir que cuando un estudiante "se pierde" en su celular en plena clase, es porque para él la clase no le resulta interesante o divertida, lo cual puede ser por varias razones no excluyentes una de otra, entre las que pueden estar las situaciones personales, expectativas de aprendizaje, dinámicas que el docente haga, el entorno, el método de transmisión de conocimiento que el docente ocupe, entre otras. El reto para los docentes es ofrecer clases presenciales o no presenciales que sean lo más atractivas posibles para que el usar el celular para asuntos no relacionados a la misma no sea una opción para el alumno”.

Estoy muy de acuerdo con que se utilice el celular para el aprendizaje por las siguientes razones:

- Brinda movilidad, flexibilidad y adaptación de los contenidos.

- Disponibilidad 24/7 de contenidos.

- Permite la interactividad inmediata o diferida entre compañeros de clase.

- Permite actividades distintas para el aprendizaje, que posiblemente no se podrían si no se usarán.

- El celular es muy apto para la gamificación (educación y aprendizaje basado en juegos). 
Lic. Domingo Alfaro, Director de Comunicaciones de la Universidad Tecnológica de El Salvador. "Claro, como recurso para acceso a la formación y de dispositivo de Power Point es importante que haya aplicaciones de conexión inalámbrico para proyectarlo en el cañón”.

Ing. Blanca Montes, integrante de la Junta Directiva del Instituto Salvadoreño de Turismo: "Sí, porque se puede generar la participación de los estudiantes en ese momento. El celular sirve para buscar hasta información en Wikipedia”.

Lic. José Amílcar Chávez Díaz, catedrático de la Universidad Tecnológica de El Salvador, se expresó así: " Sí, por supuesto que el maestro debe crear las condiciones; por ejemplo, enviarles el escrito que orientará el desarrollo de un control de lectura; o desarrollar un foro de discusión, y con su teléfono consigna su participación y en vivo les pides ampliación”.

Licenciada Carolina Márquez, catedrática de la Universidad Francisco Gavidia, manifestó: "No, rotundo... los alumnos no saben usar el dispositivo para ello. No lo usan como recurso didáctico y mucho menos de investigación. Con el celular en el aula se distraen mucho y dispersan su atención”. Los maestros tienen opiniones encontradas; por sentido común, un maestro con competencias modernas permitirá el uso de los celulares inteligentes de una forma correcta $y$ guiará a los alumnos en aprendizajes diferentes, sin olvidar el contexto de la educación.

Herberth Alexander Oliva en su investigación "El uso de teléfonos móviles en el sistema educativo público de El Salvador: ¿Recurso didáctico o distractor pedagógico?”, concluye que: "se puede señalar que en los últimos años la tecnología ha tenido grandes avances, y... la telefonía celular no ha sido la excepción... la telefonía celular está afectando el aprendizaje de los jóvenes, ya que estos muestran dispersión, falta de concentración, actitud poco reflexiva y crítica, baja calidad de las actividades...". ${ }^{7}$

El investigador que realizó las encuestas formuló dos preguntas claves: 1) ¿Han considerado proponer el uso del celular como un recurso didáctico para los estudiantes? 2) ¿Para qué utilizas el celular? Sobre la primera pregunta, el 85\% respondió que no y el resto que sí. A la segunda pregunta, los alumnos contestaron así: para hablar con la familia, para mensajear, para hablar con amigos, para revisar las redes sociales, para navegar en Internet, para escuchar música, para tomar fotografías, para jugar y como calculadora. Las respuestas no reflejan que lo utilizasen para investigar. Ver Gráfico 1.

Si su respuesta consiste en que está prohibido el uso del celular, pero a pesar de esto lo siguen usando, ¿qué hacer ante estos casos?

\begin{tabular}{|l|c|}
\hline & \% \\
\hline Se decomisa y se entrega a sus padres & $\mathbf{7 7 . 6}$ \\
\hline Se decomisa y se entrega al final del año & $\mathbf{0 . 6}$ \\
\hline Lo mandan a la Dirección & $\mathbf{5 . 0}$ \\
\hline Se decomisa y se entrega después de la jornada & $\mathbf{4 . 3}$ \\
\hline Llamado de atención & $\mathbf{1 1 . 2}$ \\
\hline El vigilante revisa a los alumnos & $\mathbf{1 . 2}$ \\
\hline \multicolumn{2}{|c|}{ Base $88.5 \%$} \\
\hline
\end{tabular}

Fuente: Centro de Estudios Ciudadanos UFG. Instituto de Ciencia, Tecnología e Innovación (ICTI - UFG).

7 Oliva, Herberth Alexander. "El uso de teléfonos móviles en el sistema educativo público de El Salvador: ¿Recurso didáctico o distractor pedagógico?” Realidad y Reflexión, Universidad Francisco Gavidia, julio diciembre 2014. 


\section{Gráfico $\mathrm{N}^{\circ} .1$}

¿Para qué cree usted que los estudiantes utilizan el celular?

Para mensajear

Para revisar las redes sociales

Para jugar

Para escuchar música

Para navegar

Para hablar con la familia

Para tomar fotografías

Para hablar con amigos

Otros
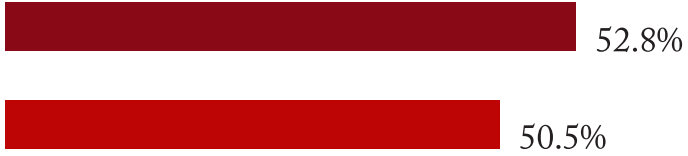

$41.2 \%$

\section{$16.5 \%$}

$14.8 \%$

$6.0 \%$

Fuente: Centro de Estudios Ciudadanos UFG. Instituto de Ciencia, Tecnología e Innovación (ICTI - UFG).

Herberth Oliva concluye que "se debe diseñar una estrategia didáctica para integrar el uso de celulares como un medio alfabetizador funcional para tecnologías". La investigación se realizó en centros escolares públicos de educación media. Los pocos estudios hechos en El Salvador sobre la utilización de los celulares en el aula son de suma importancia. Véase que la abundancia de los teléfonos inteligentes se nota en todo nivel educativo.

Encuesta realizada en cuatro universidades para investigar la utilización de smartphones en los salones de clases

Esta investigación cuantitativa se realizó, como se mencionó en la introducción, en cuatro universidades: una pública y tres privadas.

Las carreras en referencia son estas: doctorado en Medicina y licenciaturas en Mercadotecnia y Publicidad, Relaciones Públicas y Comunicaciones, Administración de Empresas, Administración de Empresas Turísticas y Contaduría Pública. 


\section{Gráfico $\mathrm{N}^{\circ} .2$}

Con respecto al género

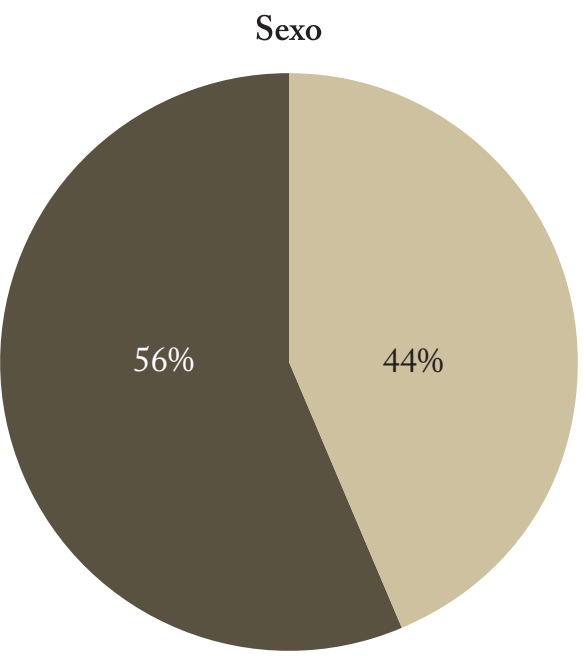

Masculino

Femenino

\section{Gráfico N. 3}

¿Por qué razón utiliza usted el celular en clases?

Objetivo: Identificar para qué utilizan los celulares los alumnos en el salón de clases.
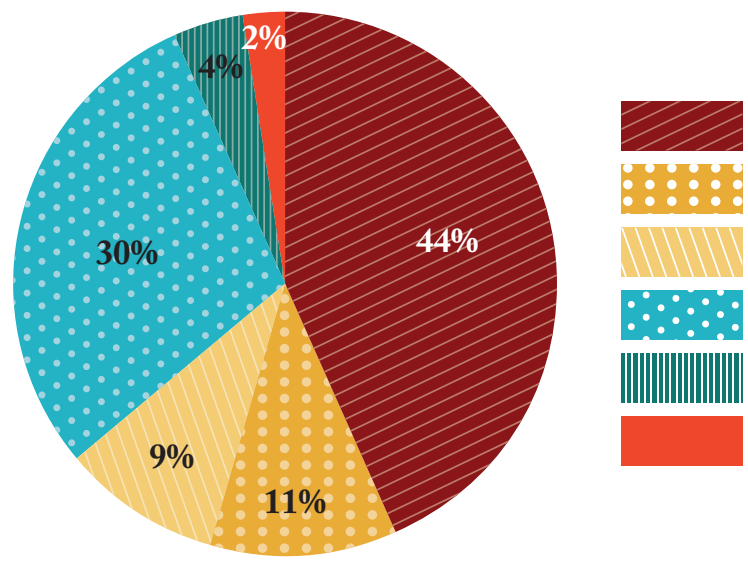

Para aprender a investigar más

La clase no me es interesante

El profesor no cumple las expectativas

Me gusta chatear y usar las redes sociales

Para jugar

Desmotivación al estudio

Análisis. Se comprueba que el 44\% de los alumnos utiliza el celular para aprender e investigar más. Aunque se verifica que en el aula, les gusta estar en un 30\% chateando y utilizando las redes sociales. En definitiva, es un distractor, el cual tiene que ser supervisado por los maestros; eso ayudará a que no afecte el rendimiento escolar de los alumnos. Y en cuanto a que la clase es aburrida, eso es una disyuntiva que debe analizarse en el sentido de que un celular es más interesante que la propia clase. 


\section{Gráfico N. ${ }^{\circ} 4$}

¿Permite el profesor utilizar celular para consultar en el salón de clases?

Objetivo: Saber si los maestros permiten que los alumnos utilicen el celular en el salón de clases.

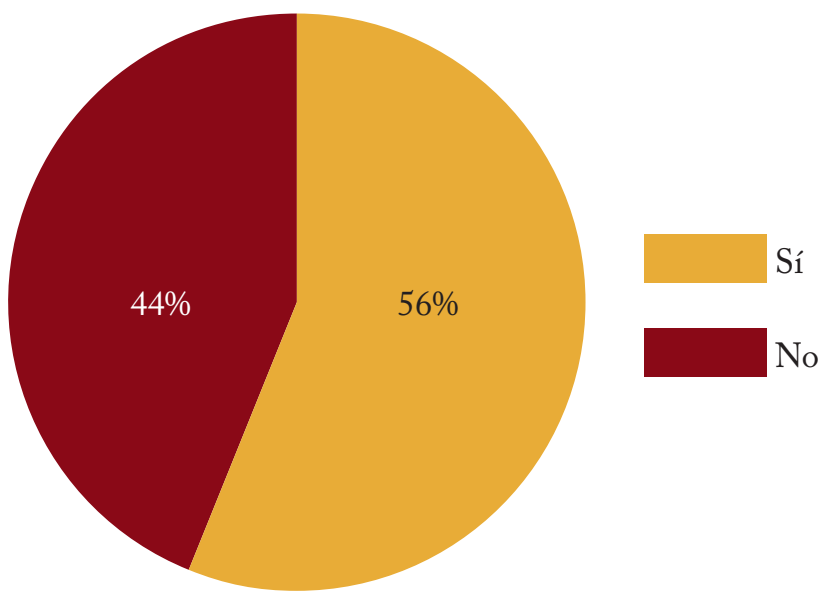

Análisis. Según los alumnos, los maestros utilizan el celular en clases. Se observa que los alumnos utilizan el celular para consultas educativas y no para otros usos.

\section{Gráfico N. ${ }^{\circ} 5$}

¿Estaría de acuerdo en que las clases se desarrollen con teléfonos inteligentes para mayor aprendizaje? Objetivo: Identificar si los alumnos están de acuerdo en que las clases se desarrollen con teléfonos inteligentes para mayor aprendizaje.

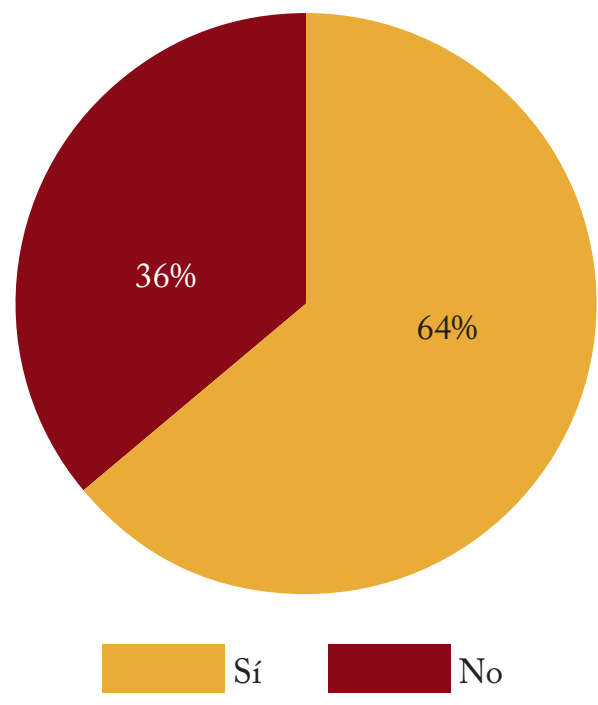

Análisis. El 64\% manifestó que está de acuerdo en que se utilicen teléfonos inteligentes en el salón de clases. Con ello, se verifica que el celular es útil siempre y cuando sea utilizado para aprender. La anterior es una interrogante clave para poder verificar que poco a poco, los maestros y alumnos están adaptándose a la utilización de los teléfonos inteligentes. 


\section{Gráfico N. ${ }^{\circ} 6$}

¿Sería más productiva una cátedra si a todos los alumnos les permitieran utilizar el teléfono inteligente para el aprendizaje?

Objetivo: Descubrir si serían productivas las cátedras si se permitiese a los alumnos utilizar su smartphone.

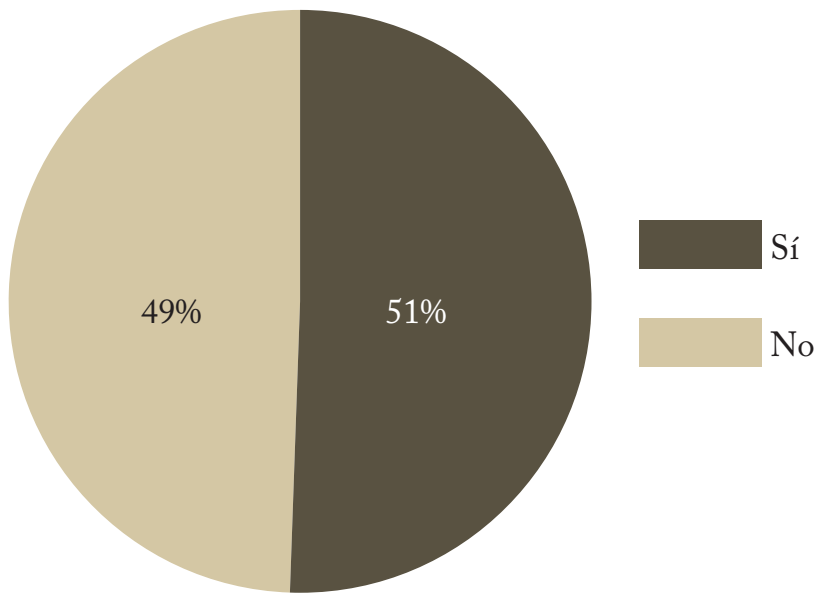

Análisis. Acá la disyuntiva radica en que si sería productiva la clase. E1 51\% manifiesta que sí; sin embargo, depende del tipo de productividad. Casi hay un emparejamiento en las opiniones, ya que los alumnos están con sus teléfonos inteligentes en el aula, pero perdiendo el tiempo y no aprovechando el conocimiento.

\section{Gráfico N. ${ }^{\circ} 7$}

¿Está de acuerdo en que el maestro utilice su propio teléfono celular para guiar la clase?

Objetivo: Investigar si los alumnos están de acuerdo en que los maestros utilicen sus propios teléfonos celulares en el salón de clases para uso didáctico.

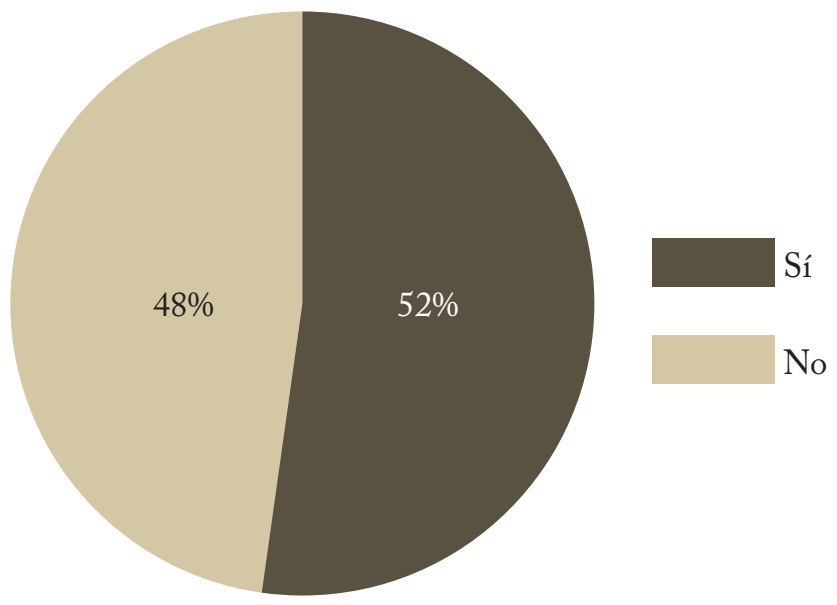

Análisis. Casi hay un empate en los resultados, lo que refleja que el $52 \%$ de los alumnos manifestó estar de acuerdo en que los maestros utilicen su propio teléfono celular para guiar la clase. Algunos maestros no lo utilizan para consultas educativas. 


\section{Gráfico N. ${ }^{\circ} 8$}

¿Mejoraría el aprendizaje de los alumnos y sería más interactiva la clase y si el maestro utilizara el teléfono smartphone para guiarla?

Objetivo: Indagar si mejoraría el aprendizaje de los alumnos, y sería más interactivo si los maestros utilizaran el celular para guiar la clase o como herramienta pedagógica.

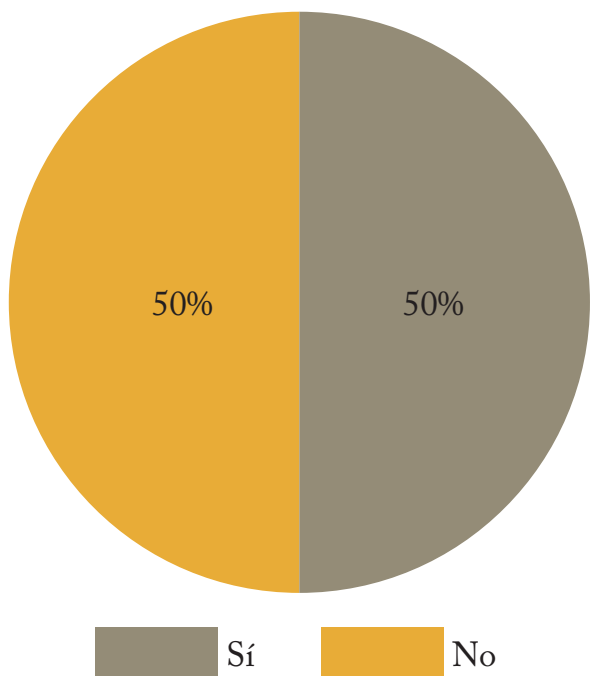

Análisis. Se comprueba que 50\% de los alumnos menciona que sería más interactiva la clase, si el maestro utilizara el teléfono para guiarla.

\section{Gráfico N. ${ }^{\circ} 9$}

¿Considera que las políticas de no usar el celular en los salones de clases son suficientes como para evitar su utilización?

Objetivo: Comprobar si las políticas de no usar celular en las clases son suficientes para evitar que los alumnos las sigan utilizando.

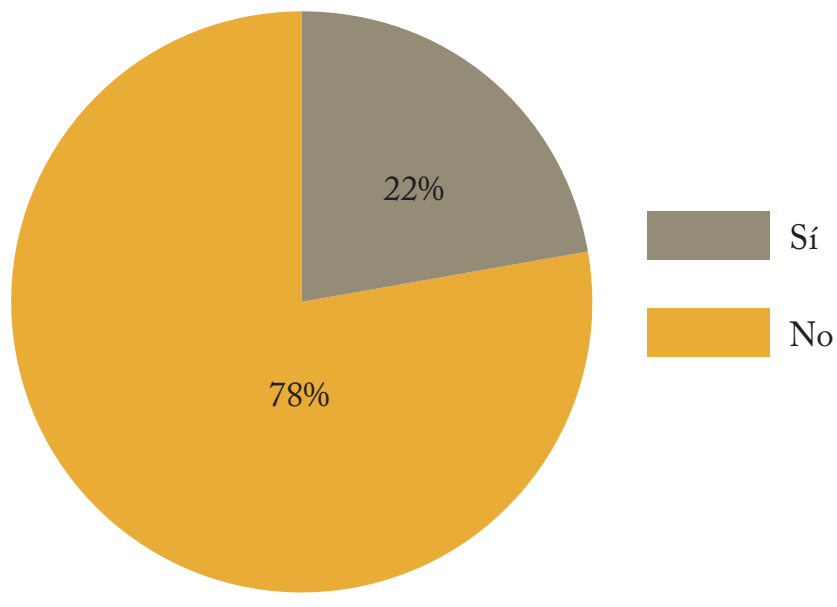

Análisis: E1 78\% considera que las políticas utilizadas por las autoridades en el uso de celulares en el aula son insuficientes. Cada universidad debe controlar el uso de los celulares en el salón de clases; sin embargo, si es para investigar y como recurso didáctico, las políticas se deben redactar acorde a usos didácticos y de investigación. 


\section{Gráfico N. ${ }^{\circ} 10$}

¿Si se permitiera a todos los alumnos el uso de celulares, qué resultados se obtendrían según su análisis? Objetivo: Analizar qué resultados se obtendrían si se dejara utilizar en las universidades el uso del celular sin restricciones.
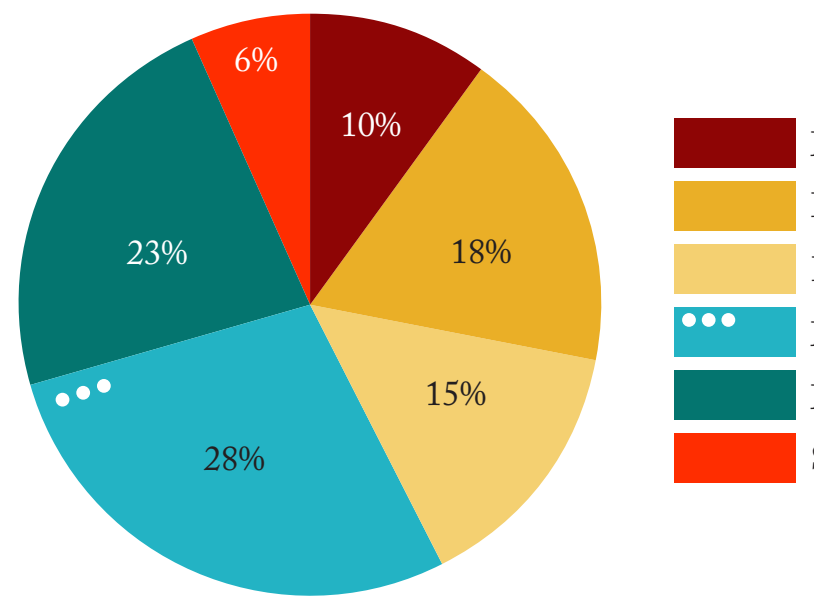

Más aprendizaje

Desorden en el salón de clases

El profesor no podría impartir las clases

Más comunicación e investigación

Más ocio de parte de los alumnos

Será un fenómeno distractor

Análisis. Se comprueba en un 33\% que hubiese más comunicación e interacción en el aula utilizando los teléfonos inteligentes, eso ayudaría al proceso de enseñanza-aprendizaje y se lograría una mayor interacción en el aula. El ocio también predomina, es usual que los alumnos que están sentados atrás del salón de clases, manifiesten poco interés en la clase que imparte el maestro.

\section{Gráfico N. ${ }^{\circ} 11$}

¿Sería constructivo que, por lo menos unos 10 minutos o más, se permitiera el uso del celular para consultas en los buscadores de su smarthphone?

Objetivo: Saber si es factible que por lo menos 10 minutos o más se utilice celular en el salón de clases.

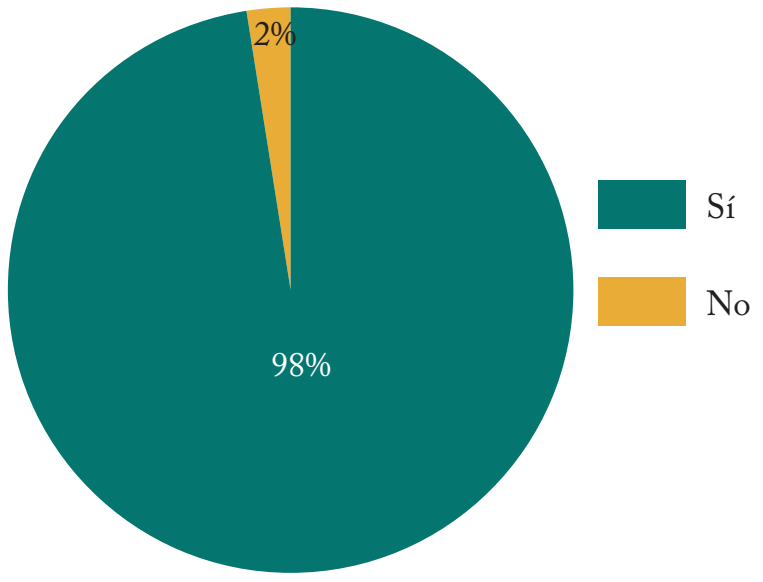

Análisis: Se comprueba que los alumnos están dispuestos a utilizar el celular por lo menos 10 minutos en el aula para el aprendizaje. E1 98\% estaría de acuerdo en consultar para aprender más y no para perder el tiempo. Mientras la clase tradicional se desarrolle normalmente, el profesor puede permitir un breve lapso de tiempo para que los alumnos indaguen sobre vocabulario técnico encontrado en la temática u otro tipo de consulta, ya sea para el aprendizaje o para investigar sobre la clase. 


\section{Gráfico N. ${ }^{\circ} 12$}

\section{¿Cuál es su opinión sobre el uso de los celulares en la clase?}

Objetivo: Identificar qué opinión tienen los alumnos al utilizar celulares en las clases.


Está sustituyendo la pedagogía tradicional

Los alumnos ponen más atención

Ayuda al constructivismo

Interrumpe la clase

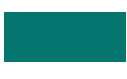

Ayuda a ser más dinámico e interactivo el proceso de aprendizaje

El celular es importante para la información no académica

Análisis. No existe un porcentaje que mencione en sí solo aspectos positivos o negativos. El 48\% manifestó que el celular interrumpe la clase. Acá, es entonces en donde el maestro tiene que poner más atención y control en la utilización de los celulares en el aula. Se comprueba entonces que las interrogantes números 8 y 10 se contradicen. Por ejemplo en la contestación a la pregunta 8, se manifiesta claramente que con el celular habría más comunicación e investigación esto, al saber que los maestros responsables tendrían cuidado en vigilar y aclarar que el celular debe utilizarse para investigar.

No es que el celular esté sustituyendo a la pedagogía tradicional; empero los alumnos desean que las clases sean interactivas y con procesos modernos de aprendizaje.

\section{Conclusiones}

En la interrogante que se realizó en la red social Facebook la mayoría de alumnos no está de acuerdo con la utilización de las redes sociales. Mientras que la minoría manifiesta que si el celular es para fines educativos, entonces debe aprovecharse con fines de investigación de la clase.

Los maestros a los que se les preguntó sobre su uso, están de acuerdo en su utilización, siempre y cuando sea para el aprendizaje de los alumnos. $\mathrm{Su}$ análisis se enfoca en el aprendizaje moderno; no se debe, entonces, prohibir en pleno siglo XXI, el celular en el salón de clases.

Si el uso de los celulares en el aula fuese controlada por los maestros, su utilización sería más eficiente.

Los docentes permiten más del 50\% el uso de los celulares en el salón de clases. Si se 
permitiese para uso académico, el aprendizaje de los estudiantes mejoraría. Los celulares interrumpen las clases; un 50\% de los alumnos consultados no desea que los maestros utilicen los celulares en el salón de clases; esto, en cierta medida se contradice con otras interrogantes; ya que, los maestros responsables, utilizarían los teléfonos inteligentes para investigar en el aula. Aseveración que se percibe en el salón de clases. No solamente el libro físico se puede utilizar en la actualidad. Los maestros utilizan las tecnologías educativas para enseñar mejor.

Los alumnos consultados mencionaron que las políticas para su utilización no son suficientes. Los avisos en carteles con la advertencia de "No use el celular", son una censura; sin embargo, las autoridades universitarias, deben de evaluar el hecho de que en la actualidad casi todos los alumnos poseen un celular. La utilización de estos en aprendizajes en el salón de clases, se mezcla entre textos, mensajes, redes sociales y otros.

Los alumnos investigados están de acuerdo con que por lo menos unos diez minutos los maestros permitan la utilización de los celulares en el salón de clases.

Más comunicación e investigación serían los resultados al utilizar los smartphones. Según la encuesta, empero esa comunicación debe ser supervisada. Si hay constructivismo en el aula, los maestros pueden formularle una pregunta a un alumno que posea un teléfono inteligente.

Mientras la clase tradicional se desarrolle normalmente, el profesor puede permitir un breve lapso de tiempo para que los alumnos indaguen sobre vocabulario técnico encontrado en la temática u otro tipo de consulta para el aprendizaje. Se observa que en los salones de clases, hay docentes que no tienen ningún control sobre el uso de los celulares, lo que puede influir en el bajo rendimiento del alumno. Por lo tanto, un breve tiempo para utilizar el celular, si es permitido, podría generar más conocimiento y aprendizaje entre los discentes.

En definitiva, el celular viene a ser un distractor, por lo que tiene que ser supervisado por los maestros. Si no hay control acerca de su uso, ello afectará el rendimiento escolar de los alumnos. El celular, pues, se tiene que utilizar estrictamente para investigación.

Mientras más se utilicen los celulares, de manera adecuada, los alumnos van perdiendo hasta adquirir ortografía.

El celular acrecienta el ocio y contribuye a las malas notas y a la distracción en la clase. Si se tomase el ejemplo del video de YouTube, en donde el maestro acomoda el salón de clases, o sea, lo hace dinámico y logra estar al unísono con los jóvenes de la "Generación Y".

A manera de recomendación, es de analizar si las autoridades universitarias están o no de acuerdo en limitar el uso de los celulares en el aula. Desde el momento en que se entra al salón de clases, se ven alumnos con uno o dos aparatos sobre el pupitre. Si lo utilizan para consultar temas, actividades u otra información relacionada con la clase, se debe permitir el celular; si no debe prohibirse.

Los maestros deben comprender que no estarán durante determinada cátedra regañando o 
pendientes de quiénes están conectados a páginas educativas en el salón de clases. Todo el mundo sabe que la "U Virtual" o plataforma educativa puede estar siendo visitada por los alumnos; además, pueden estar viendo la clase, la cual han descargado en el celular. Lo idóneo y constructivista sería que, el docente lance una interrogante y que participen los alumnos con sus teléfonos inteligentes con el propósito de responder a la pregunta.

La investigación hecha por este autor constata que los alumnos desean tener el permiso para utilizar el celular, pero para comunicarse y hacer investigación. Por lo tanto, se debe permitir el uso del celular en cierta medida y dependiendo de la asignatura. Al final se evaluará si el uso de los teléfonos inteligentes es favorable o no para el proceso de enseñanza-aprendizaje.

\section{Bibliografía}

- Franco, J. (2008). Educación y Tecnología: solución radical. México: Siglo XXI, p. 290
- Heredia, Y.y Martínez, R. (2010). Tecnología educativa en el salón de clase. Estudio retrospectivo de su impacto en el desempeño académico de estudiantes universitarios del área de Informática. Revista Mexicana de Investigación Educativa, 15 (45). Recuperado de: http://www.redalyc.org/articulo. oa?id=14012507003

- Oliva, H. (2014, julio-diciembre). E1 uso de teléfonos móviles en el sistema educativo público de El Salvador: ¿Recurso didáctico o distractor pedagógico? Revista Realidad y Reflexión, Universidad Francisco Gavidia, E1 Salvador.

\section{Sitios web}

- ht t p s:// w w w youtube.com / watch?v=rZOXT_MUlzE

- http://www.leanstart.es/millennials-unanueva-generacion/

- http://www.abcbilingualschool.edu.sv/Inicio/ 
Anexo

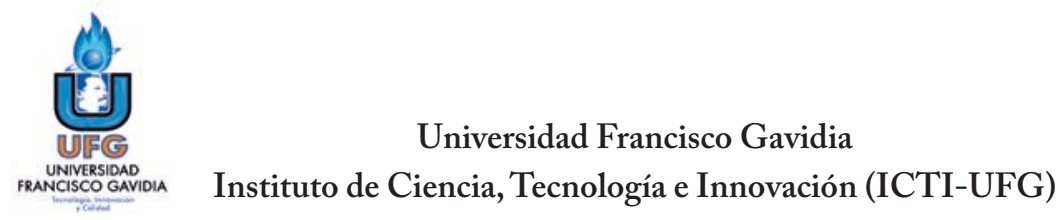

Docente Investigador: MEd. Fidel Arturo López Eguizábal

Investigación: La generación de estudiantes y maestros que utilizan los smartphones y otras tecnologías educativas en las aulas universitarias para mejorar el proceso de la enseñanza aprendizaje.

Objetivo: Indagar sobre los efectos positivos y negativos de la utilización de tecnologías educativas, en especial el smartphones, en las aulas universitarias.

Indicación: Por favor marque con una "X" su opción de preferencia en cada numeral.

Sexo: Masculino $\square$ Femenino $\square$

1. ¿Por qué razón utiliza usted el celular en clases?

a) Para aprender e investigar más.

b) La clase no me es interesante.

c) El profesor no cumple las expectativas.

d) Me gusta chatear y usar las redes sociales.

e) Para jugar.

f) Desmotivación al estudio.



2. ¿Permite el profesor utilizar celular para consultas en el salón de clases?

Sí

No

3. ¿Estaría de acuerdo en que las clases se desarrollen con teléfonos inteligentes para mayor aprendizaje? Sí

No

4. ¿Sería más productiva una cátedra si a todos los alumnos se les permitiera utilizar el teléfono inteligente para el aprendizaje?

Sí

No 
5. ¿Está de acuerdo en que el maestro utilice su propio teléfono celular para guiar la clase?

Sí

No

6. ¿Mejoraría el aprendizaje de los alumnos y sería más interactiva la clase si el maestro utiliza el teléfono smartphone para guiar el tema de la clase?

Sí

No

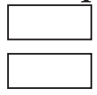

7. ¿Considera que las políticas de no usar celular en los salones de clases son suficientes como para evitar su utilización?

Sí

No

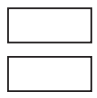

8. ¿Si se permitiera a todos los alumnos el uso de celulares qué resultados, según su análisis, se obtendrían?

a) Más aprendizaje.

b) Desorden en el salón de clases.

c) El profesor no podría impartir las clases.

d) Más comunicación e investigación.

e) Más ocio de parte de los alumnos.

f) Sería un fenómeno distractor.

9. ¿Sería constructivo que por lo menos unos 10 minutos o más se permitiera el uso del celular para consultas en los buscadores?

Sí

No

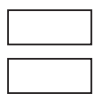

10. ¿Cuál es su opinión sobre el uso de los celulares en la clase?

a) Está sustituyendo la pedagogía tradicional.

b) Los alumnos ponen más atención.

c) Ayuda al constructivismo.

d) Interrumpe la clase.

e) Ayuda a ser más dinámico e interactivo el proceso educativo.

f) El celular es importante para información no académica. 